\title{
B-LEARNING Y E-LEARNING COMO ESTRATEGIAS PARA EL DESARROLLO DE COMPETENCIAS COMPLEMENTARIAS DEL ALUMNADO DEL GRADO DE MAESTRO
}

\author{
Marcos Gómez-Puerta \\ marcos.gomez@ua.es \\ Gonzalo Lorenzo Lledó \\ glledo@ua.es \\ Graciela Arráez Vera \\ Graciela.arraez@ua.es \\ Alejandro Lorenzo Lledó \\ alejandro.Iorenzo@ua.es \\ Facultad de Educación \\ Universidad de Alicante
}

\section{RESUMEN}

La sobrecarga de contenidos curriculares en la educación superior puede hacernos pasar por alto determinadas competencias que resultan clave para la formación y el futuro ejercicio profesional del alumnado universitario. En este sentido, iniciativas como los NOOC pueden servirnos para complementar la formación de los estudiantes mediante el desarrollo en profundidad de determinados contenidos y/o capacidades. Determinadas universidades están impulsando el desarrollo de estas iniciativas mediante la puesta en marcha de convocatorias de innovación docente. En este trabajo se presenta un ejemplo de NOOC para el desarrollo de mejores competencias de atención a la diversidad y desarrollo de una educación inclusiva por parte de estudiantes de los grados de Maestro en Educación Infantil y Primaria de la Facultad de Educación de la Universidad de Alicante.

Palabras clave: NOOC; educación superior; formación a distancia; complementos de formación

\section{ABSTRACT \\ B-learning and e-learning as strategies for the development of complementary competences of the teaching degree students \\ The overload of curricular contents in higher education can make us overlook certain compe- tences that are key to the training and future professional practice of university students. In this sense, initiatives such as Nano Online Open Courses (NOOC) can complement the training of stu- dents through the in-depth development of certain content and / or skills. Certain universities are}




\section{B-LEARNING Y E-LEARNING COMO ESTRATEGIAS PARA EL DESARROLLO DE COMPETENCIAS COMPLEMENTARIAS DEL ALUMNADO DEL GRADO DE MAESTRO}

promoting the development of these initiatives through the implementation of calls for teaching innovation. This paper presents an example of NOOC for the development of better skills in attention to diversity and development of an inclusive education by students of the Teaching degrees in Early Childhood and Primary Education of the Faculty of Education of the University of Alicante.

Keywords: NOOC; higher education; distance learning; training complements

\section{ANTECEDENTES}

Las nuevas tecnologías de la información y la comunicación han abierto un espectro enorme de posibilidades en diversos ámbitos, entre los que se encuentra la educación. De ellos, la formación a distancia (e-learning) o la formación semipresencial (b-learning) son dos de las tendencias que parecen tener un potencial desarrollo más prometedor en el ámbito de la educación superior (Roig, Mengual y Rodríguez, 2013).

Diversas universidades han puesto en marcha iniciativas para el desarrollo de Massive Online Open Courses (MOOC) o bien Nano Online Open Courses (NOOC) con el fin de incentivar la puesta en marcha de estas nuevas perspectivas de formación en línea. En concreto, la Universidad de Alicante (UA) a través del Vicerrectorado de Calidad e Innovación Educativa y en colaboración con el Instituto de Ciencias de la Educación ha realizado convocatorias para impulsar dichas actuaciones en los años 2014, 2016 y 2017. En concreto, la última convocatoria se denominó Convocatoria de ayudas a proyectos de innovación educativa para la promoción de la enseñanza semipresencial y online (Programa Pensem-online)(Boletín Oficial de la Universidad de Alicante, 10.11.2017). El programa PENSEM-ONLINE pretende la promoción de la enseñanza a distancia, a través de cursos concretos ofrecidos por el profesorado, o bien mediante la incorporación de este tipo de enseñanza en titulaciones oficiales de Grado y Máster. Este programa es una de las acciones de innovación educativa enmarcadas en el Programa Institucional Innovación, Investigación, Internacionalización y Colaboración en Educación (I3CE), 2016-2020, del Instituto de Ciencias de la Educación de la UA. Esta línea de innovación pone de manifiesto por parte de la UA compromiso hacia la calidad y la innovación educativa en la Educación Superior. Asimismo, esta convocatoria se aseguraba la formación continua del profesorado universitario puesto que todos los participantes en la convocatoria quedaban obligados a participar de un curso de formación específicos para el desarrollo de contenidos multimedia online, en línea con las propuestas realizadas por Vásquez y Sevillano (2011).

Los fines de esta convocatoria fueron, por una parte, difundir la enseñanza no presencial o semipresencial en la UA mediante la creación y difusión de MOOC y NOOC y, por otra parte, ayudar a las diversas facultades en la implementación de docencia tanto a distancia como semipresencial en las titulaciones oficiales vigentes. Las líneas prioritarias establecidas abarcaban los siguientes posibles enfoques:

Cursos orientados a facilitar el acceso a titulaciones oficiales o que pueda motivar a cursar una titulación oficial.

Cursos que puedan utilizarse como complemento de capacidades específicas no cubiertas en una titulación oficial.

Cursos introductorios, o cursos "cero", que se adaptarán al procedimiento y la normativa de la UA establecida para los cursos de formación que le sea aplicable.

Cursos para complementar la formación sobre salidas profesionales de las titulaciones y mejora de la empleabilidad.

Cursos de formación para personal docente e investigador orientados a la mejora de la docencia universitaria.

Cursos dirigidos en general a la sociedad para difundir el conocimiento generado en la UA. 
En el caso de la iniciativa que aquí se presenta, se decidió explorar la segunda línea, la específicamente ligada a complementar determinadas capacidades específicas no cubiertas suficientemente en una titulación oficial. En nuestra reflexión como grupo de trabajo abordamos la necesidad de fomentar un mayor desarrollo del conocimiento del alumnado de los Grados de Maestro de Educación Infantil y de Educación Primaria respecto de cómo desarrollar proyectos educativos inclusivos, directamente derivados de la competencia específica CE7 que se aplica a varias asignaturas de dichos estudios. En concreto esta competencia a desarrollar consiste en:

CE7: Diseñar y desarrollar proyectos educativos, unidades de programación, entornos, actividades y materiales, incluidos los digitales, que permitan adaptar el currículum a la diversidad del alumnado y promover la calidad de los contextos en los que se desarrolla el proceso educativo, de modo que se garantice su bienestar.

No obstante, en nuestra experiencia considerábamos que dicha competencia podía no desarroIlarse con suficiente amplitud y profundidad por lo que decidimos abordar un NOOC como estrategia para complementar el desarrollo de esta capacidad específica en asignaturas como Gestión e innovación en contextos educativos, Atención a necesidades educativas específicas, o Atención a discapacidades sensoriales y motrices. En concreto, determinamos que el Index for inclusion (Booth y Ainscow, 2002) podía resultar una buena estrategia para que el alumnado pudiese completar su formación para adquirir las capacidades específicas relacionadas con la mencionada competencia.

Como respuesta a las necesidades detectadas se planificó un NOOC de 18 horas de duración, que se prolongaría a lo largo de tres semanas, y orientado específicamente al alumnado del Grado de Maestro de Infantil o Primaria. Éste se desarrollaría a través de la plataforma Moodle de la UA.

\section{OBJETIVOS}

Los objetivos del curso se concretaron en:

Objetivo general: conocer el Index for Inclusion y su aplicación como instrumento para la mejora de la educación inclusiva en los centros escolares.

Objetivos específicos:

Conocer los elementos que configuran una educación de calidad desde la perspectiva de la inclusión.

Conocer y analizar los elementos que configuran el Index for Inclusion.

Utilizar las tres dimensiones que configuran el Index for Inclusion como herramientas para la evaluación y mejora de la inclusión en un centro escolar.

\section{PARTICIPANTES}

El contenido del curso se orienta al alumnado de los grados de Maestro de Educación Infantil y Educación Primaria. No obstante, el curso presenta un carácter abierto, por lo que personas de otros estudios u alumnado ajeno a nuestra universidad podrían acceder a dicha acción formativa.

\section{MÉTODO}

Respecto a la estructura y contenidos del NOOC, se acordó desarrollar los siguientes objetivos y contenidos modulares:

Módulo 1. Introducción a la estructura y funcionamiento del curso.

Objetivo: explicar los objetivos del curso, contenidos que se van a tratar, la metodología que se va a seguir, así como el sistema de evaluación previsto. Asimismo, se realizará una evaluación inicial de conocimientos previos.

Contenidos:

Estructura del curso: objetivos, contenidos, actividades, temporalización. 
¿Qué sabes ya sobre este tema?

Duración prevista: 1.5 horas (primera semana).

Módulo 2. La educación inclusiva: claves para su desarrollo.

Objetivo: conocer los elementos que configuran una educación de calidad desde la perspectiva de la inclusión.

Contenidos:

Historia de la Educación Especial: breve repaso histórico.

La educación inclusiva.

Diseño para todos, accesibilidad universal y su relación con el Universal Learning Design (UDL). Duración prevista: 4.5 horas (primera semana).

Módulo 3. El Index for inclusion como herramienta para la mejora de la educación inclusiva.

Objetivo: conocer y analizar los elementos que configuran el Index for Inclusion.

Contenidos:

El Index for Inclusion como guía para la evaluación y mejora de la educación inclusiva.

Dimensiones del Index for inclusion.

Etapas en el proceso de trabajo con el Index for inclusion.

Indicadores y preguntas para la evaluación.

Duración prevista: 6 horas (segunda semana).

Módulo 4: la utilización práctica del Index for Inclusion

Objetivo: Utilizar las tres dimensiones que configuran el Index for Inc/usion como herramientas para la evaluación y mejora de la inclusión en un centro escolar.

Contenidos:

Aplicación de la dimensión 1: crear culturas inclusivas.

Aplicación de la dimensión 2: elaborar políticas inclusivas.

Aplicación de la dimensión 3: desarrollar prácticas inclusivas.

Duración prevista: 6 horas (tercera semana).

\section{Metodología del curso}

El curso está organizado en 4 módulos, siendo el primero de ellos introductorio. El curso comienza con un módulo introductorio relativo a la organización y funcionamiento del mismo. Este módulo contiene un vídeo de presentación así como una guía didáctica del curso. Además, se realiza un cuestionario sobre los conocimientos previos del alumnado.

El resto de módulos comparten una estructura común: presentación del módulo, contenidos estructurados, actividades, evaluación, recursos de aprendizaje, recursos y actividades complementarios, foro. Todos los módulos incluyen material audiovisual (vídeos, podcast, presentaciones).

Los contenidos del curso se orientan eminentemente a su uso práctico, favoreciendo que el alumnado adquiera competencias para la implementación de éstas en su futuro ejercicio profesional. Las actividades favorecen el aprendizaje autónomo y la autoevaluación del alumnado mediante la comparación de sus respuestas con un patrón de respuesta elaborado por los docentes. El alumnado comparte en un foro las dudas y los materiales que se diseñan, generándose así un aprendizaje colaborativo.

La duración prevista del curso es de tres semanas, estimándose una carga de trabajo para el alumnado de 18 horas (1.5, 4.5, 6 y 6 horas de dedicación para cada módulo, respectivamente). Se ha calculado que la dedicación semanal aproximada es de 6 horas.

\section{Actividades formativas}

El tipo de actividades previstas en el desarrollo del NOOC son las siguientes: 
Visionado del material audiovisual.

Lectura y análisis de los materiales presentados: videos tutoriales, documentos y enlaces.

Realización de las prácticas de cada módulo. Corrección de las mismas según los indicadores 0 patrones proporcionados por el equipo docente.

Realización del cuestionario de evaluación de cada módulo.

Participación en el foro grupal a través de consultas y aportaciones a las preguntas planteadas.

Realización de la encuesta sobre calidad del curso y satisfacción con el mismo.

\section{Recursos empleados}

En todos los módulos del curso se utilizarán los siguientes recursos:

Introducción al módulo. Vídeo con la presentación del tema, los objetivos de aprendizaje y contenidos, así como la secuencia temporal orientativa de trabajo.

Desarrollo de los contenidos del módulo. Material audiovisual y documental presentado.

Evaluación. Cuestionario de evaluación de aprendizajes.

Recursos básicos. Carpeta con material básico del módulo (documentación videos, artículos, enlaces web).

Recursos complementarios. Material y actividades de profundización en el tema, en el caso de que el alumnado quiera ampliar su formación (documentación videos, artículos, enlaces web).

Foro de participación y debate.

Cuestionario de evaluación de la calidad del curso y satisfacción con el mismo.

\section{RESULTADOS}

En este curso se está realizando la primera promoción del NOOC señalado, por lo que no se dispone aún de una evaluación sumativa de los resultados logrados con el mismo, incluyendo la percepción y satisfacción con el mismo del profesorado y alumnado. No obstante, resulta una iniciativa motivadora para el profesorado y alumnado, y que presenta visos de un mayor desarrollo futuro.

La evaluación se realizará atendiendo a diversos criterios: (1) el número de horas que el alumno/a ha permanecido conectado a la plataforma del curso (se establecerá un mínimo), (2) la realización y entrega de las actividades en el plazo establecido, (3) la participación activa en el foro de cada módulo (al menos una aportación por módulo), y (4) la realización y superación del cuestionario de evaluación del módulo. Asimismo, se analizará la calidad del curso y la satisfacción del alumnado con el mismo mediante la recogida de respuestas a un cuestionario diseñado ad hoc.

\section{CONCLUSIONES}

La sobrecarga de contenidos curriculares en la educación superior puede hacernos pasar por alto determinadas competencias que resultan clave para la formación y el futuro ejercicio profesional del alumnado universitario. En este sentido, iniciativas como los NOOC pueden servirnos para complementar la formación de los estudiantes mediante el desarrollo en profundidad de determinados contenidos y/o capacidades. Determinadas universidades, como por ejemplo la Universidad de Alicante, están impulsando el desarrollo de estas iniciativas mediante la puesta en marcha de convocatorias de innovación docente.

\section{REFERENCIAS BIBLIOGRÁFICAS}

Booth, T. y Ainscow, M. (2002). Guía para la evaluación y mejora de la educación inclusiva. Desarrollando el aprendizaje y la participación en las escuelas. Madrid: CSIE y Consorcio Universitario para la Educación Inclusiva. 
Roig, R., Mengual, S., y Rodríguez, C. (2013). Internet como medio de información, comunicación y aprendizaje. En J. Barroso y J. Cabero (Coords.), Nuevos escenarios digitales. Las tecnologías de la información y la comunicación aplicadas a la formación y desarrollo curricular (pp. 209234). Madrid. Pirámide.

Vázquez, E. y Sevillano, M.L. (2011). Educadores en red. Elaboración y edición de materiales audiovisuales para la enseñanza. Madrid: UNED/Ediciones Académicas. 